\title{
Baixas doses de óxido nítrico na seleção dos pacientes candidatos a transplante cardíaco com hipertensão pulmonar
}

\author{
Juan Alberto Cosquillo MEJIA*, Valdester Cavalcante PINTO Jr. *, Haroldo Brasil BARROSO*, \\ Fernando Antônio MESQUITA*, Waldomiro CARVALHO Jr.*, José Matos Brito CASTELO \\ BRANCO**, Flávio José Rocha SOUZA*, Maria Corina Amaral VIANA*, Patrícia Lopes SOUSA*, \\ João David SOUSA NETO*
}

RBCCV 44205-527

Mejia J A C, Pinto Jr V C, Barroso H B, Mesquita F A, Carvalho Jr W, Castelo Branco J M B, Souza F J R, Viana M C A, Sousa P L, Sousa Neto J D - Baixas doses de óxido nítrico na seleção dos pacientes candidatos a transplante cardíaco com hipertensão pulmonar. Rev Bras Cir Cardiovasc 16(1): 28-34.

RESUMO: A disfunção do ventrículo direito no pós-operatório do transplante cardíaco é uma complicação freqüente com morbimortalidade elevada. A avaliação hemodinâmica pulmonar dos candidatos precisa, às vezes, do emprego de prova farmacológica com o uso de drogas endovenosas vasodilatadoras tipo nitroprussiato de sódio (NTPNa), visando reduzir os níveis de pressão e resistência vascular pulmonar (RVP) elevados a níveis compatíveis com os protocolos de inclusão dos Programas de Transplante Cardíaco. Valores de RVP acima de 6 unidades Wood sem droga vasodilatadora, ou acima de 2,5 com a utilização destas, excluem os pacientes do Programa. Entre janeiro de 1997 e janeiro de 2000 foram submetidos a cateterismo direito, 40 pacientes candidatos a transplante. A maioria homens, com idade mé ( 12 anos). As etiologias mais encontradas foram miocardiopatia dilatada idiopática (59\%) e miocardiopatia isquêmica (25\%). Todos em CF IV da NYHA, com fração de ejeção média do ventrículo esquerdo de 0,21 ( 0,03$)$. A monitorização do débito cardíaco (DC), pressão da artéria pulmonar (PAP) e RVP foi feita pelo método contínuo, utilizando para isto cateter de artéria pulmonar especial. Em 5 pacientes a prova farmacológica foi interrompida devido aos efeitos sistêmicos do NTPNa com queda da PAM, RVP e DC. Todos foram submetidos a inalação de óxido nítrico (NO) por máscara, a uma dose de 20 ppm durante 10 minutos. Em 3, a RVP reduziu de $7,8(0,88)$ para $2,4(0,36)$ unidades Wood, e eles foram incluídos no Programa de Transplante. Nos outros 2, apesar de doses de NO de 20,30 e 40 ppm, a redução foi de $8,4(2,12)$ para $4,9(0,42)$ unidades Wood, ou seja, insuficiente para serem incluídos nos Programas de Transplante. Não tivemos óbitos, nem complicações durante os procedimentos. O NO inalatório na avaliação hemodinâmica dos candidatos a TC - pelo seu efeito vasodilatador seletivo - permite identificar aqueles com hiper-resistência vascular pulmonar, que não respondem ao uso de drogas vasodilatadoras endovenosas convencionais, evitando que sejam excluídos dos benefícios do procedimento do TC.

DESCRITORES: Óxido nítrico, administração \& dosagem. Vasodilatadores, administração \& dosagem. Transplante de coração. Hipertensão pulmonar. Disfunção ventricular direita, prevenção \& controle. Resistência vascular. Cuidados pré-operatórios. Transplante cardíaco, cuidados pré-operátorios.

\footnotetext{
Trabalho realizado no Hospital do Coração de Messejana I e no Hospital Antônio Prudente. Fortaleza, CE, Brasil. Apresentado no $26^{\circ}$ Congresso Nacional de Cirurgia Cardíaca. Fortaleza, CE, 8 a 10 de abril de 1999.

* Do Hospital do Coração de Messejana.

** Do Hospital Antônio Prudente.

Endereço para correspondência: Juan Alberto Cosquillo Mejia. Clínica Cirucárdio. Rua Vicente Nogueira Braga, 160. Fortaleza, CE, Brasil. CEP: 60040-570. Tel: (85) 272-8580. e-mail: cirucard@daterranet.com.br.
} 
Mejia J A C, Pinto Jr V C, Barroso H B, Mesquita F A, Carvalho Jr W, Castelo Branco J M B, Souza F J R, Viana M C A, Sousa P L, Sousa Neto J D - Baixas doses de óxido nítrico na seleção dos pacientes candidatos a transplante cardíaco com hipertensão pulmonar. Rev Bras Cir Cardiovasc 16(1): 28-34.

\section{INTRODUÇÃO}

Apesar de avanços importantes na melhora da sobrevida dos transplantados cardíacos tenham sido conseguidos com progressos referentes à terapia imunossupressora, técnicas cirúrgicas, métodos de avaliação da rejeição aguda e crônica, cuidados intensivos pós-operatórios, entre outros; a disfunção do ventrículo direito no pós operatório imediato é ainda um acontecimento freqüente $(50 \%$ de todas as complicações), mantendo-se como uma das principais causas de mortalidade precoce (até $19 \%$ em algumas séries) (1). Esforços têm sido feitos para identificar àqueles pacientes candidatos à transplante com hipertensão pulmonar severa e hiper-resistência vascular pulmonar fixa, que não respondem à administração de drogas vasodilatadoras endovenosas, tipo nitroprussiato de sódio (NTPNa), já que estes pacientes fazem parte do grupo de risco que pode apresentar a referida complicação, o que colocaria em perigo, não só a vida do paciente, bem como a vida útil do enxerto a curto prazo. Na prática, uma elevada resistência vascular periférica (RVP) maior que 6 a 8 unidades Wood é, geralmente, considerada uma contra-indicação ao transplante cardíaco (2).

O óxido nítrico (NO) é um gás que, quando administrado de forma inalatória, se comporta como um vasodilatador pulmonar seletivo. O NO dentro do alvéolo se difunde rapidamente através da membrana alvéolo-capilar provocando o relaxamento da musculatura lisa do vaso pulmonar (Figura 1). À medida que se difunde dentro da luz do vaso sangüíneo é fixado pela hemoglobina e inativado. A afinidade da hemoglobina pelo NO é três mil vezes

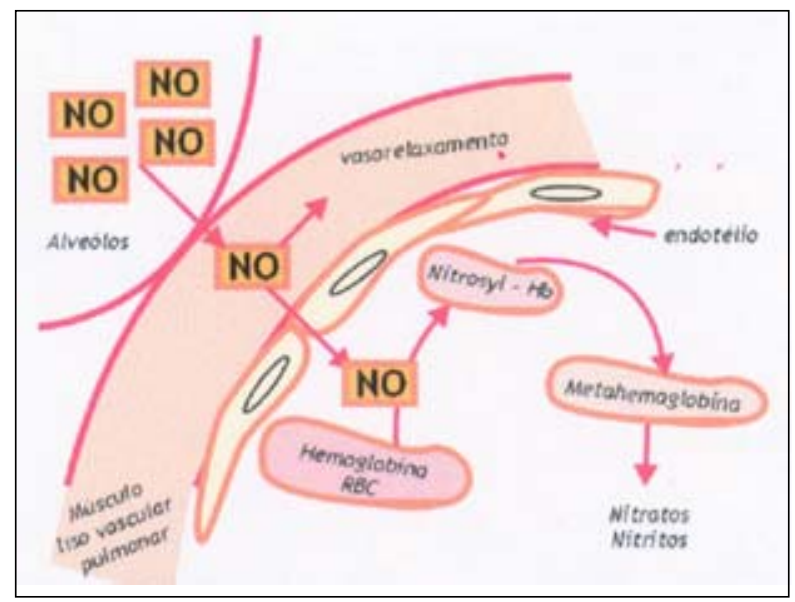

Fig. 1 - Modo de ação do NO inalatório. Após produzir vasodilatação pulmonar, o NO é inativado rapidamente pela hemoglobina dos eritrócitos (RCB)

Nitrosyl-hb: Nytrozil-hemoglobina maior que pelo oxigênio. Quando fixada ao NO, a hemoglobina é convertida em nitrosyl-hemoglobina, e logo após em meta-hemoglobina. A metahemoglobina se converte em nitratos e nitritos pela meta-hemoglobina reductase que se encontra nos eritrócitos. A maior parte dos nitratos e nitritos circulantes no sangue se produzem a partir do NO endógeno. Em razão da sua união à hemoglobina, as propriedades do NO inalado estão localizadas clinicamente na circulação pulmonar, sem produzir vasorelaxamento sistêmico indesejado. A ação vasodilatadora do NO é interrompida quando este é retirado do circuito ventilatório. Desta forma, os efeitos farmacológicos do NO são eliminados com a interrupção da droga (3).

O presente trabalho avaliou a resposta da administração do NO inalatório em baixas doses (20ppm) em pacientes onde não foi possível reverter a hiper-resistência vascular pulmonar usando drogas vasodilatadoras convencionais, tipo NTPNa.

\section{CASUÍSTICA E MÉTODOS}

Entre janeiro de 1997 e janeiro de 2000 foram estudados para avaliação hemodinâmica pulmonar 40 pacientes candidatos a transplante cardíaco, sendo $30 \%$ do sexo feminino com idade média de 42 anos ( 12 anos); todos em CF NYHA IV, fração de ejeção média: $0,21(0,03)$ e no máximo com insuficiência tricuspídea leve; a etiologia mais encontrada foi a miocardiopatia dilatada idiopática - 24 casos $(59 \%)$, seguida por miocardiopatia isquêmica -10 casos (25\%), miocardiopatia chagásica - 3 casos (8\%), miocardiopatia periparto - 2 casos $(5 \%)$, miocardiopatia pós-viral - 1 caso (3\%) (Gráfico 1).

O cateterismo direito foi realizado no laboratório de hemodinâmica com o paciente acordado, através de punção preferencialmente da veia jugular interna direita pelo método de Seldinger, e na presença de

\section{GRÁFICO 1}

\section{ETIOLOGIA. DISTRIBUIÇÃO POR ETIOLOGIAS: 40 PACIENTES}

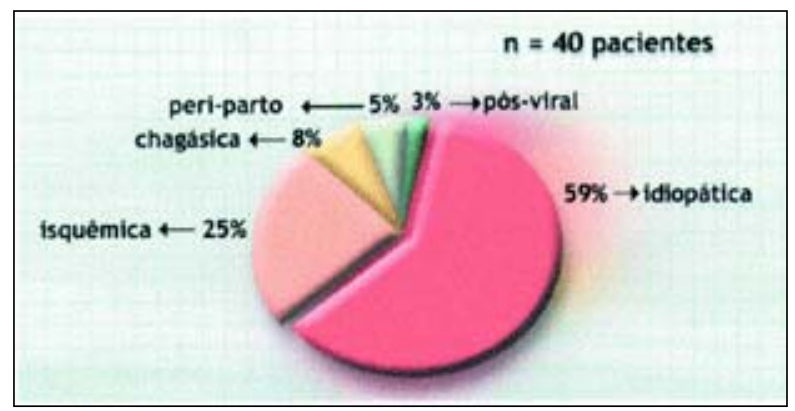


Mejia J A C, Pinto Jr V C, Barroso H B, Mesquita F A, Carvalho Jr W, Castelo Branco J M B, Souza F J R, Viana M C A, Sousa P L, Sousa Neto J D - Baixas doses de óxido nítrico na seleção dos pacientes candidatos a transplante cardíaco com hipertensão pulmonar. Rev Bras Cir Cardiovasc 16(1): 28-34.

um anestesiologista. Utilizamos para isso cateter de artéria pulmonar com balão direcionado pelo fluxo, com filamento de aquecimento distal (Modelo 744 - 7.5 Fr. BAXTER, EDWARDS SWAN GANZ . CCO/SVO2. hemodilution catheter Heparin Coated) (Figura 2), que permite o registro do Débito Cardíaco (DC) e cálculo de resistências de forma quase contínua ${ }^{(4,5)}$, com intervalos de aproximadamente 30 segundos (Figura 3), num Debímetro BAXTER VIGILANCE (Figura. 4). A pressão arterial média sistêmica (PAM) era registrada através de um cateter $5 \mathrm{Fr}$ colocado por punção numa das artérias femurais.

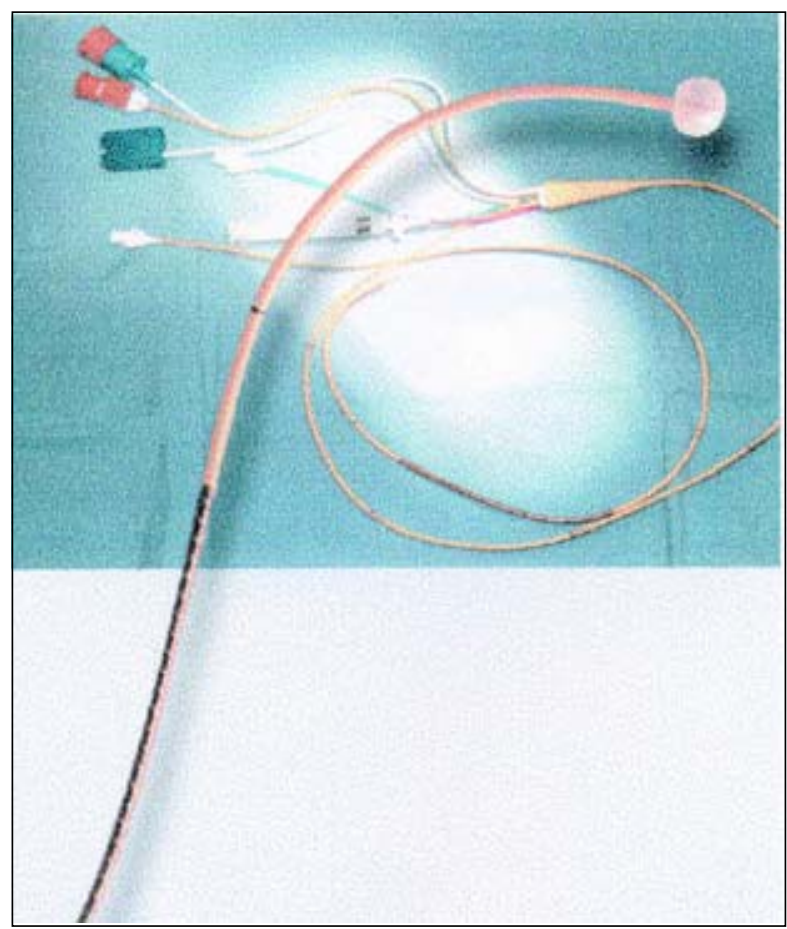

Fig. 2 - Detalhe do cateter da artéria pulmonar, com filamentos de aquecimento distal

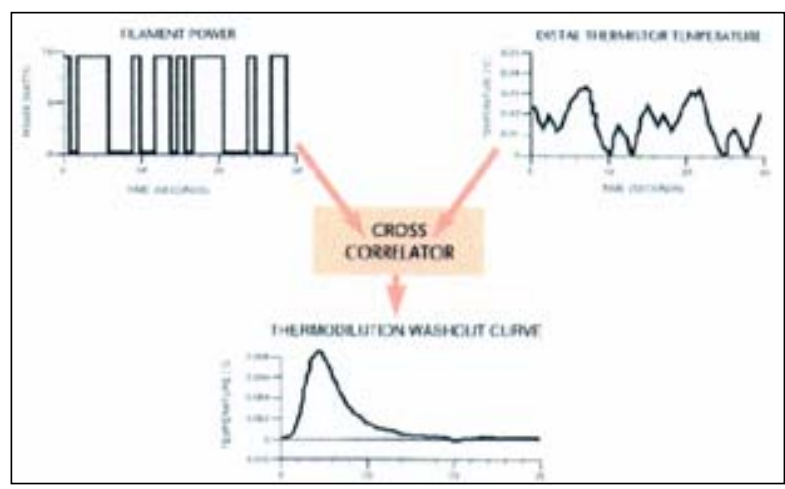

Fig. 3 - Diagrama mostrando a corelação entre os impulsos elétricos produzidos no filamento do cateter de Swan Gans, as mudanças de temperatura captadas no thermistor distal e a curva de terma diluição gerada
Quando resistência vascular pulmonar (RVP) era maior que 6 unidades Wood, fazia-se prova farmacológica com doses crescentes de NTPNa endovenoso (começando com doses baixas de 25 $\mathrm{mcg} / \mathrm{min}$ ), até obter uma pressão sistólica da artéria pulmonar menor que $40 \mathrm{mmHg}$ e/ou RVP menor ou igual a 2,5 unidades Wood, e se eventualmente o NTPNa provocava queda de resistência vascular sistêmica (RVS), com PAM menor que $65 \mathrm{mmHg}$ ou queda do DC, suspendia-se a prova ${ }^{(6)}$, (Gráfico 2) e instalava-se circuito inalatório de NO por máscara facial com presilha tipo Downs (EPAP- Expiratory Positive Airway Preasure, sem válvula de pressão expiratória) (Figura 5) a doses de 20 ppm, com uma mistura de oxigênio a 50\%, por 10 minutos (7) antes de fazer novos registros. O NO é fornecido através de cilindros (Figura 6) com volume de $3 \mathrm{~m}^{3}$ a uma concentração de 500 ppm (WHITE MARTINS), e a monitorização de $\mathrm{NO}$ e $\mathrm{NO}_{2}$ produzidos é feita através de um analisador composto por uma célula eletroquímica.

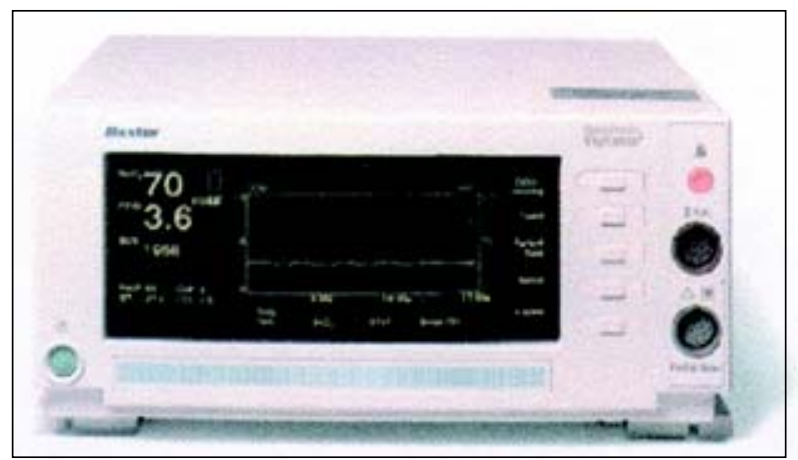

Fig. 4 - Debimetro BAXTER ${ }^{\star *}$ VIGILANCE para monitorização continua do débito cardíaco

\section{GRÁFICO 2}

FLUXOGRAMA DE AVALIAÇÃO HEMODINAMICA PULMONAR DOS CANDIDATOS A TRANSPLANTE CARDÍACO

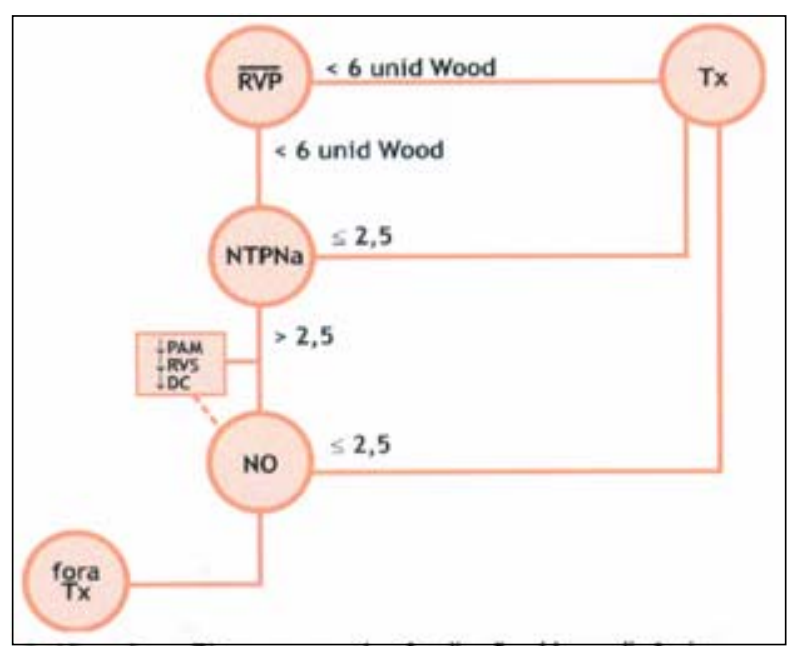


Mejia J A C, Pinto Jr V C, Barroso H B, Mesquita F A, Carvalho Jr W, Castelo Branco J M B, Souza F J R, Viana M C A, Sousa P L, Sousa Neto J D - Baixas doses de óxido nítrico na seleção dos pacientes candidatos a transplante cardíaco com hipertensão pulmonar. Rev Bras Cir Cardiovasc 16(1): 28-34.

TABELA 1

DOS VALORES MÉdICOS DE RVP E DC ANTES E APÓS ADMINISTRAÇÃO DE NO INALATÓRIO

\begin{tabular}{lccccc}
\hline \multirow{2}{*}{ Total 5 pacientes } & \multicolumn{2}{c}{ Pró-No } & \multicolumn{2}{c}{ Pós-No } & resultados \\
\cline { 2 - 5 } & RVP & DC & RVP & DC \\
3pts-NO & 7,8 unid/Wood & $2,0 \mathrm{~L} / \mathrm{min}$ & 2,4 unid/Wood & $3,0 \mathrm{~L} / \mathrm{min}$ & incluídos prog. \\
$(20 \mathrm{ppm})$ & $(+-0,88)$ & $(+-0,34)$ & $(+-0,2)$ & & \\
$2 \mathrm{pts}-\mathrm{NO}$ & 8,4 unid/Wood & $1,8 \mathrm{~L} / \mathrm{min}$ & 4,9 unid/Wood & $2,2 \mathrm{~L} / \mathrm{min}$ & excluídos prog. \\
$(20 / 30 / 40 \mathrm{ppm})$ & $(+-2,12)$ & $(+-56)$ & $(+-) 0,42)$ & $(+-0,28)$ & \\
\hline
\end{tabular}

NO: Óxido Nítrico; RVP: Resistência Vascular Pulmonar; DC: Débito Cardíaco

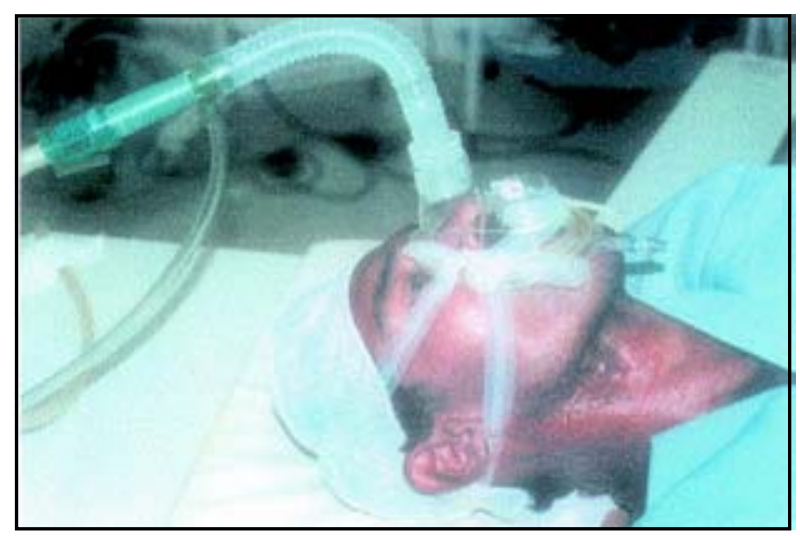

Fig. 5 - Circuito inalatório de NO por máscara facial

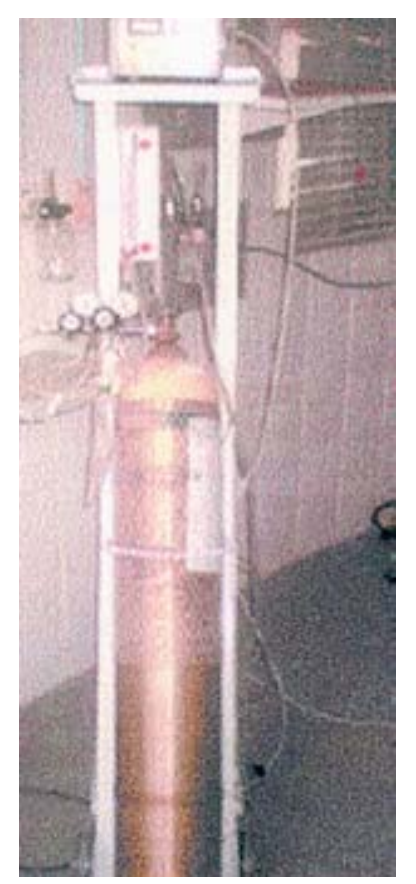

Fig. 6 - Sistema de administração do NO, cilindro, conexões e na parte superior o analisador de $\mathrm{NO}$ e $\mathrm{NO}_{2}$

\section{RESULTADOS}

Dos 40 pacientes estudados, 35 foram incluídos no programa de transplante cardíaco com ou sem prova farmacológica com NTPNa. Em 3 pacientes foram utilizados NO (20ppm); a RVP média pré NO, era de $7,8(0,88)$ unidades Wood e foi para 2,4 $(0,36)$ unidades Wood pós NO. O DC médio aumentou de 2,0 $(0,34) \mathrm{L} / \mathrm{min}$ pré $\mathrm{NO}$, e foi para 3,0 $(0,2) \mathrm{L} / \mathrm{min}$ pós NO. E com estes resultados, estes pacientes também foram incluídos no Programa. Nos 2 pacientes restantes, foram utilizados $\mathrm{NO}(20,30 \mathrm{e}$ $40 \mathrm{ppm}) ;$ a RVP média pré NO era de $8,4(2,12)$ unidades Wood e foi para 4,9 $(0,42)$ unidades Wood pós NO. O DC médio aumentou de $1,8(0,56) \mathrm{L} /$ min pré NO, e foi para $2,2(0,28) \mathrm{L} / \mathrm{min}$ pós NO. Segundo o protocolo em vigência, estes pacientes foram excluídos do Programa de Transplante Cardíaco Ortotópico (Tabela 1).

Nenhum dos pacientes estudados foi a óbito, assim como, nenhum deles apresentou edema agudo do pulmão ou comprometimento grave do estado hemodinâmico que indicasse a suspensão da prova com NO inalatório por este motivo.

Destes pacientes que foram submetidos à avaliação hemodinâmica pulmonar, 14 foram transplantados, 3 se submeteram a cirurgias alternativas para

\section{GRÁFICO 3}

RESULTADOS. DISTRIBUIÇÃO DOS CANDIDATOS À TRANSPLANTE CARDÍACO SUBMETIDOS Á AVALIAÇÃO HEMODINAMICA

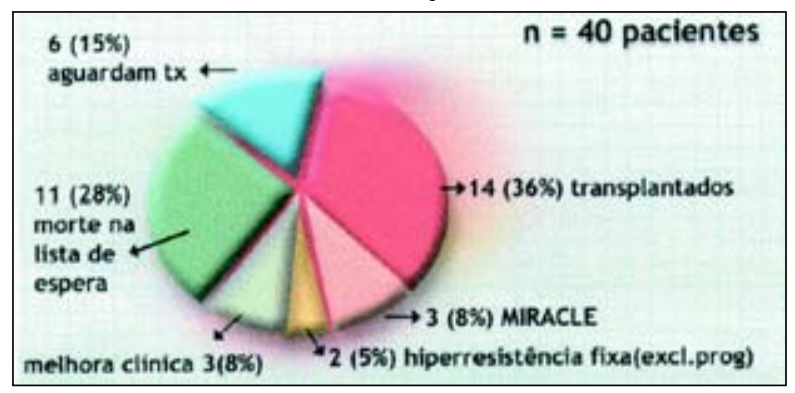


Mejia J A C, Pinto Jr V C, Barroso H B, Mesquita F A, Carvalho Jr W, Castelo Branco J M B, Souza F J R, Viana M C A, Sousa P L, Sousa Neto J D - Baixas doses de óxido nítrico na seleção dos pacientes candidatos a transplante cardíaco com hipertensão pulmonar. Rev Bras Cir Cardiovasc 16(1): 28-34.

reparo de disfunção secundária da válvula mitral (MIRACLE), 2 não entraram no Programa por hiperresistência fixa, 3 saíram da lista de espera por melhora clínica, 11 morreram aguardando doação, e os 6 restantes aguardam doadores para serem transplantados (Gráfico 3).

\section{COMENTÁRIOS}

A disfunção do ventrículo direito no pós-operatório imediato do transplante cardíaco continua sendo uma preocupação constante nos centros transplantadores em razão de corresponder a uma porcentagem elevada dentro das complicações encontradas (até $50 \%$ ), e que pode provocar uma alta mortalidade precoce $(19 \%)$ ou uma diminuição da vida útil do enxerto. Esforços devem ser feitos nos laboratórios de hemodinâmica para identificar aqueles pacientes com hipertensão pulmonar severa $e$ com hiper-resistência vascular pulmonar fixa, que não respondem às provas farmacológicas com drogas vasodilatadoras pulmonares, tais como NTPNa e prostaglandina $E 1{ }^{(8)}$. O efeito sistêmico destas drogas que provoca queda na RVS, na PAM e, algumas vezes no DC, evita que se obtenha uma diminuição das pressões e das resistências da artéria pulmonar a níveis suficientes para que os pacientes avaliados sejam incluídos nos programas de transplante, segundo os protocolos vigentes.

O NO inalatório tem se mostrado eficaz no tratamento de situações associadas a quadros de hipertensão pulmonar, tais como no pós-operatório de cirurgias cardíacas com circulação extracorpórea, no tratamento da disfunção do ventrículo direito pós transplante cardíaco, no peri-operatório do transplante pulmonar, na síndrome de distress respiratório do adulto, na hipertensão pulmonar persistente do recém-nascido, entre outras ${ }^{(9-15)}$.

Estudos têm demonstrado que o NO quando utilizado em baixas doses (até 80 ppm) não prejudica a função ventricular esquerda, e esta carência de efeito pode ser, em parte, pela rápida inativação do NO livre em trânsito ao coração dos pacientes avaliados (16,17). KIELER-JENSEN et al. (18) em 1994 utilizando $\mathrm{NO}$ em doses crescentes de 20, 40 e 80 ppm na avaliação dos candidatos a transplante com hipertensão pulmonar severa, comprovou que este era inócuo e que poderia ser administrado sem riscos aos pacientes. ADATIA et al. (19) em 1995 estudou 11 pacientes com hipertensão pulmonar $\mathrm{e}$ candidatos a transplante e demonstrou que o NO, além de ser um potente vasodilatador pulmonar com efeito sistêmico mínimo, também poderia ser útil para diferenciar aqueles pacientes que precisariam de transplante cardiopulmonar daqueles que teriam indicação apenas para transplante cardíaco ortotópico.
Entretanto, BOCCHI et al. (20) em 1994 apontaram a possibilidade de edema pulmonar após a utilização de NO durante a avaliação hemodinâmica pulmonar em 3 candidatos a transplante, com doses de 40 a 80 ppm; e atribuíram isto a uma redução aguda da pós carga do ventrículo direito, com grande aumento de retorno sanguíneo a um ventrículo esquerdo altamente comprometido, que não consegue ejetar esta sobrecarga de volume, provocando aumento do capilar pulmonar e edema agudo de pulmão.

$O$ registro do DC contínuo permite perceber pequenas alterações hemodinâmicas em intervalos curtos de tempo, o que facilita a decisão sobre as mudanças das doses das drogas vasodilatadoras empregadas, bem como uma eventual suspensão do procedimento ante a leitura de uma tendência negativa, o que poderia provocar um sério desequilíbrio hemodinâmico que colocaria em risco a vida do paciente. Além disso, este registro contínuo, ao reduzir o tempo total do procedimento, diminui também o tempo do paciente na posição horizontal, se comparado a outros métodos de registro do DC, o que é muito importante em se tratando de pacientes gravemente comprometidos (21).

Neste trabalho, administramos o NO inalatório para aqueles pacientes que não responderam de forma satisfatória ao uso de uma droga vasodilatadora endovenosa convencional (NTPNa), já que estes não atingiram o nível mínimo necessário de pressão e de resistência vascular pulmonar que possibilitasse o seu ingresso ao programa de transplante. $O$ fato de que, em 3 dos 5 pacientes estudados, conseguíssemos quedas de pressão e de resistência pulmonares, a níveis admissíveis segundo os protocolos de inclusão, demonstra a possibilidade de poder identificar estes pacientes que de outra forma, pelo método convencional, seriam excluídos do programa.

\section{CONCLUSÕES}

O emprego do NO inalatório, pelo seu efeito vasodilatador pulmonar seletivo, na avaliação hemodinâmica dos pacientes candidatos a transplante cardíaco, permite identificar aqueles pacientes com hiper-resistência vascular pulmonar severa, que não respondem ao uso de drogas vasodilatadoras convencionais, tipo NTPNa, evitando que este grupo seja excluído dos benefícios do Programa de Transplante Cardíaco. Acreditamos que, durante o emprego do NO, a utilização de uma monitorização contínua do DC ajuda a detectar precocemente a probabilidade de desequilíbrios hemodinâmicos importantes, que poderiam ter efeitos deletérios para o paciente. 
Mejia J A C, Pinto Jr V C, Barroso H B, Mesquita F A, Carvalho Jr W, Castelo Branco J M B, Souza F J R, Viana M C A, Sousa P L, Sousa Neto J D - Baixas doses de óxido nítrico na seleção dos pacientes candidatos a transplante cardíaco com hipertensão pulmonar. Rev Bras Cir Cardiovasc 16(1): 28-34.

RBCCV 44205-527

Mejia J A C, Pinto Jr V C, Barroso H B, Mesquita F A, Carvalho Jr W, Castelo Branco J M B, Souza F J R, Viana M C A, Sousa P L, Sousa Neto J D - Low doses of nitric oxide in the selection of candidates to heart transplantation with pulmonary hypertension. Rev Bras Cir Cardiovasc 2001; 16(1): 28-34.

ABSTRACT: The right ventricle dysfunction in the post-operative period of the heart transplantation (HTx) surgery is a frequent complication with high morbidity and mortality. In order to reduce the high pulmonary vascular resistance (PVR) and pulmonary artery pressure (PAP), often found in the patients candidates to $\mathrm{HTx}$, it is, sometimes, necessary to use endovenous infusion of sodium nitroprusside (NaNTP) (the pharmacologic test), during the pre-operative hemodynamic assessment of the pulmonary circulation, in attempt to reduce the PVR and PAP to compatible levels, allowing the inclusion of these patients in the HTx program protocol. Values of PVR over 6 Wood units without drugs (such as NaNTP) or 2.5 Wood units with drugs will exclude the patient from the HTx program. Between January 1997 and January 2000,40 patients (70\% males) (mean age $=42+/-12)$, candidates to HTx were submitted to cardiac catheterization (right side). 24 patients (59\%) had dilated idiopathic cardiomyopathy, 10 (25\%) ischemic myocardiopathy. All were in Class IV (NYHA), with mean left ventricular ejection fraction (LVEF) of 0.21 (+/- 0.03). Continuous measurement of the cardiac output, PAP and RVP were done with the help of a special Swan-Ganz catheter. In 5 patients the pharmacologic test was interrupted owing to systemic effects of NaNTP (important drop of arterial blood pressure and cardiac output). Then, they were submitted to inhalation of $20 \mathrm{ppm}$ of nitric oxide (NO), though facial mask, during 10 minutes, in three patients the RVP dropped from a mean of $7.8(+/-0.88)$ to $2.4(+/-0.36)$ Wood units and so they could be included in the transplantation program. The two other patients did not achieve RVP drops enough to be candidates to HTx, once, even though the amount of NO inhaled had reached 20, 30 and $40 \mathrm{ppm}$, the reduction of the RVP was from $8,4(+/-2.12)$ to $4,9(+/-0.42)$ Wood units. There were no deaths or complications during these producers. The NO inhalation during hemodynamic evaluation of candidates to HTx, owing to its selective vasodilator effect, allows us to identify those patients with pulmonary vascular hyperresistance, who do not respond to the use of endovenous vasodilator drugs, thus avoiding them to be excluded from the benefits of the HTx.

DESCRIPTORS: Nitric oxide, administration \& dosage. Vasodilator agents, administration \& dosage. Heart transplantation. Hypertension, pulmonary. Ventricular dysfunction, right, prevention \& control. Vascular resistance. Preoperative care. Heart transplantation preoperative care.

\section{REFERÊNCIAS BIBLIOGRÁFICAS}

1 Kaye M P - The Registry of the International Society for Heart and Lung Transplantation: tenth official report. J Heart Lung Transplant 1993; 12: 541-8.

2 Wahlers T H, Beer C, Fieguth H G et al. - Right heart failure following orthotopic cardiac transplantation. Clin Transplant 1988; 2: 252-6.

3 Fullerton D A \& Mclntyre R C Jr - Inhaled nitric oxide: therapeutic applications in cardiothoracic surgery. Ann Thorac Surg 1996; 61: 1856-64.

4 Haller M, Zollner C, Briegel J, Forth H - Evaluation of a new continuous thermodilution cardiac output monitor in critically ill patients: a prospective criterion standard study. Crit Care Med 1995; 23: 860-6.

5 Munro H, Wood C, Taylor B, Smith GB - Continuous invasive cardiac output monitoring: the Baxter / Edwards Critical-care Swan Ganz Intellicath and Vigilance system. Clin Intens Care 1994; 5: 52-5.

6 Dias da Silva M A, Correia E B, Magalhães H M - Heart transplantation recipients selection: indication and contraindication criteria. Rev Soc Cardiol Estado de São Paulo 1995; 6: 614-9.
7 Azeredo C A - Pressão positiva expiratória. In: Azeredo CAC. Fisioterapia respiratória moderna. São Paulo: Manole, 1993: 49-78.

8 McCarthy P M \& Stinson E B - Routine posttransplant procedures and early postoperative problems after cardiac transplantation. In: Smith JÁ, McCarthy, et al (eds). The Stanford manual of cardiopulmonary transplantation. New York: Futura Publishing Company, Inc, 1996: 63-78.

9 Riedel B - The pathophysiology and management of perioperative pulmonary hypertension with specific emphasis on the period following cardiac surgery. Int Anesthesiol Clin 1999; 37: 55-79.

10 Bender K A, Alexander J A, Enos J M, Skimming J W - Effects of inhaled nitric oxide in patients with hypoxemia and pulmonary hypertension after cardiac surgery. Am J Crit Care 1997; 6: 127-31.

11 Nonami $Y$ - The role of nitric oxide in cardiac surgery. Surg Today 1997; 27: 583-92.

12 Bichel T, Spahr-Schopfer I et al. - Successful weaning from cardiopulmonary bypass after cardiac surgery using inhaled nitric oxide. Paediatr Anaesth 1997; 7: 335-9. 
Mejia J A C, Pinto Jr V C, Barroso H B, Mesquita F A, Carvalho Jr W, Castelo Branco J M B, Souza F J R, Viana M C A, Sousa P L, Sousa Neto J D - Baixas doses de óxido nítrico na seleção dos pacientes candidatos a transplante cardíaco com hipertensão pulmonar. Rev Bras Cir Cardiovasc 16(1): 28-34.

13 Bacha E A \& Head C A - Use of inhaled nitric oxide for lung transplantation and cardiac surgery. Respir Care Clin N Am 1997; 3: 521-36.

14 Auler Jr. J O C, Carmona M J et al. - Low doses of inhaled nitric oxide in heart transplant recipients. $J$ Heart Lung Transplant 1996; 15: 443-50.

15 Ferreira E \& Shalansky S J - Nitric oxide for ARDS: what is the evidence? Pharmacotherapy 1999; 19: 60-9.

16 Troncy E, Francoeur M, Blaise G - Inhaled nitric oxide: clinical applications, indications, and toxicology. Can J Anaesth 1997; 449: 973-88.

17 Hayward C S, Kalnins W V, Rogers P, Feneley M P, MacDonald P S, Kelly R P - Effect of inhaled nitric oxide on normal human left ventricular function. $J A m$ Coll Cardiol 1997; 30: 49-56.

18 Kieler-Jensen N, Ricksten S E, Stengvist $O$ et al. -

Inhaled nitric oxide in the evaluation of heart transplant candidates with elevated pulmonary vascular resistance. J Heart Lung Transplant 1994; 13: $366-75$.

19 Adatia I, Perry S, Landzberg M, Moore P, Thompson J $E$, Wessel D L - Inhaled nitric oxide and hemodynamic evaluation of patients with pulmonary hypertension before transplantation. J Am Coll Cardiol 1995; 25: 1656-64.

20 Bocchi E A, Bacal F, Auler Júnior J O, Carmone M J, Bellotti G, Pileggi F - Inhaled nitric oxide leading to pulmonary edema in stable severe heart failure. $\mathrm{Am}$ J Cardiol 1994; 74: 70-2.

21 Mihaljevic J, von Segesser L K, Tonz M et al. - Continuous versus bolus thermodilution cardiac output measurements: a comparative study. Crit Care Med 1995; 23: 944-9. 